\title{
Systolic anterior motion of the mitral valve associated with acute type A aortic dissection: a case report describing one-step surgery of ascending aortic replacement and interventricular septal myectomy
}

\author{
Naoki Yamamoto* ${ }^{*}$ and Koji Onoda
}

\begin{abstract}
Background: Systolic anterior motion of the mitral valve associated with acute type A aortic dissection is rare in daily clinical practice. The prevention of systolic anterior motion is important, because once it occurs, the hemodynamics may become unstable, leading to a critical situation. In the surgical procedure to treat systolic anterior motion, the prevention of new iatrogenic aortic intimal tears is important in the context of acute type A aortic dissection.

Case presentation: We present a case of systolic anterior motion in a 68-year-old woman with an acute type A aortic dissection and suspected acute relative adrenal insufficiency. Preoperative transthoracic echocardiography revealed left ventricular outflow tract obstruction due to systolic anterior motion without left ventricular hypertrophy and interventricular septal bulging due to a narrow aorto-mitral angle. We successfully performed a one-step surgery for ascending aortic replacement and interventricular septal myectomy using the needle stick technique for the treatment of systolic anterior motion.

Conclusions: Concomitant interventricular septal myectomy using the needle stick technique with thoracic aortic replacement is a safe and feasible technique. Interventricular septal myectomy may be effective in preventing postoperative unstable hemodynamics due to systolic anterior motion in the management of acute aortic dissection.
\end{abstract}

Keywords: Ascending aortic dissection, Interventricular septal myectomy, Relative adrenal failure, Systolic anterior motion

\section{Background}

Systolic anterior motion (SAM) of the mitral valve is a cardiac functional disorder that can be induced by multifactorial dynamic components, such as left ventricular (LV) structures, mitral valvular or subvalvular structures, and patients' cardiovascular hemodynamics [1-4]. Preventing SAM is important, because once SAM occurs, the hemodynamics may be unstable, leading to a critical situation [5]. Here, we present a rare case of a patient with unstable SAM associated with acute type A aortic dissection (AAD) who underwent a one-step surgery for ascending aortic replacement and concomitant interventricular septal myectomy (IVSM) for the treatment of SAM.

*Correspondence: naokiyan0930@yahoo.co.jp

Department of Cardiovascular Surgery, Shingu Municipal Medical Center

18-7 Hachibuse, Shingu, Wakayama 647-0072, Japan 


\section{Case presentation}

A 68-year-old woman (body weight, $48 \mathrm{~kg}$; height, $158 \mathrm{~cm}$ ) was referred to our emergency department for acute-onset chest and back pain. On arrival, her vital signs were as follows: respiratory rate, 17 breaths/min; temperature, $36.4{ }^{\circ} \mathrm{C}$; blood pressure, 94/56 $\mathrm{mmHg}$; heart rate, 73 beats/min; oxygen saturation level, $99 \%$ in ambient air; and Glasgow Coma Scale score 15. Physical examination revealed no heart murmur and normal respiratory sounds. A 12-lead electrocardiogram showed no evidence of acute coronary syndrome. Her medical history included Stanford type B aortic dissection, hypertension, and rheumatoid arthritis controlled with anti-rheumatic drugs and alternate-day oral corticosteroids (prednisolone $5 \mathrm{mg}$ per dose). Computed tomography $(\mathrm{CT})$ revealed thrombosed AAD from the aortic root to the renal arteries, no aortic rupture, and no malperfusion. The ascending aortic maximum short diameter was $39 \mathrm{~mm}$, and the maximum thickness of the thrombosed false lumen was $4 \mathrm{~mm}$ (Fig. 1). Transthoracic echocardiography (TTE) revealed no evidence of cardiac tamponade, aortic valve insufficiency, or other significant valve diseases. Her vital signs were stable; therefore, an urgent operation at a later date for AAD was planned. Preoperative blood pressure control was performed in our intensive care unit, in combination with bed rest, to prevent recurrent aortic events. Her systolic blood pressure was maintained between 90 and $110 \mathrm{mmHg}$ with continuous infusion of a calcium antagonist (nicardipine $0.3-1.2 \mu \mathrm{g} / \mathrm{kg} / \mathrm{min}$ ). Her heart rate was between 80 and 100 beats/min, and oxygen saturation was $98 \%$ at $2 \mathrm{l} / \mathrm{min}$ oxygenation with a nasal cannula.

A day after the onset of aortic dissection, cardiac auscultation revealed a Levine $2 / 6$ systolic murmur at the apex with no diastolic component. Subsequent investigation using TTE led to the detection of SAM of the mitral valve. TTE revealed LV hypovolemia, LV outflow tract (LVOT) obstruction due to SAM without LV hypertrophy (LVH), and interventricular septal (IVS) bulging due to a narrow aorto-mitral angle of $106.4^{\circ}$ (Fig. 2a, b). The basal IVS thickness was $18.1 \mathrm{~mm}$. The LV wall motion was hyperkinetic, with an ejection fraction of $81.7 \%$. The peak flow velocity in the LVOT was $6.25 \mathrm{~m} / \mathrm{s}$. Abnormal laboratory data included hyponatremia (serum Na: $115.9 \mathrm{mEq} / \mathrm{l}$ ), and her urine output per day was approximately $3000 \mathrm{ml}$. An adrenocorticotropic hormone stimulation test revealed suspected relative adrenal failure due to physical stress after acute aortic dissection. The systolic heart murmur due to SAM tended to improve with heart rate control to between 60 and 70 beats/min with

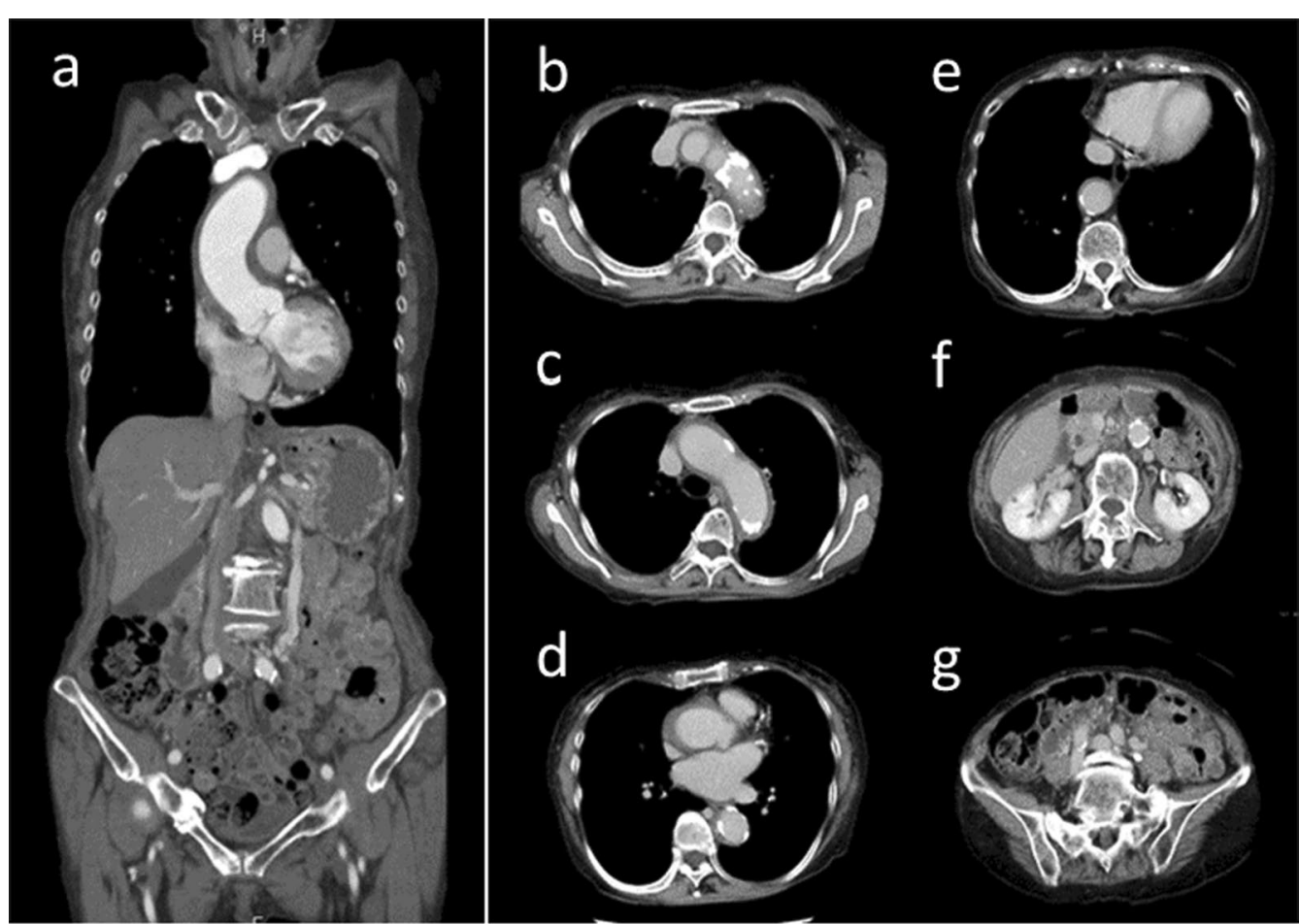

Fig. 1 Computed tomography imaging of acute type A aortic dissection. a Dissected ascending aorta. The maximum short diameter of the ascending aorta was $39 \mathrm{~mm}$, and the maximum thickness of the thrombosed false lumen was $4 \mathrm{~mm}$. b, $\mathbf{c}$ Dissected aortic arch. $\mathbf{d}$ Dissected aortic root and descending aorta of left atrial level. e Dissected descending aorta. f Non-dissected infrarenal aorta. g Non-dissected iliac arteries 


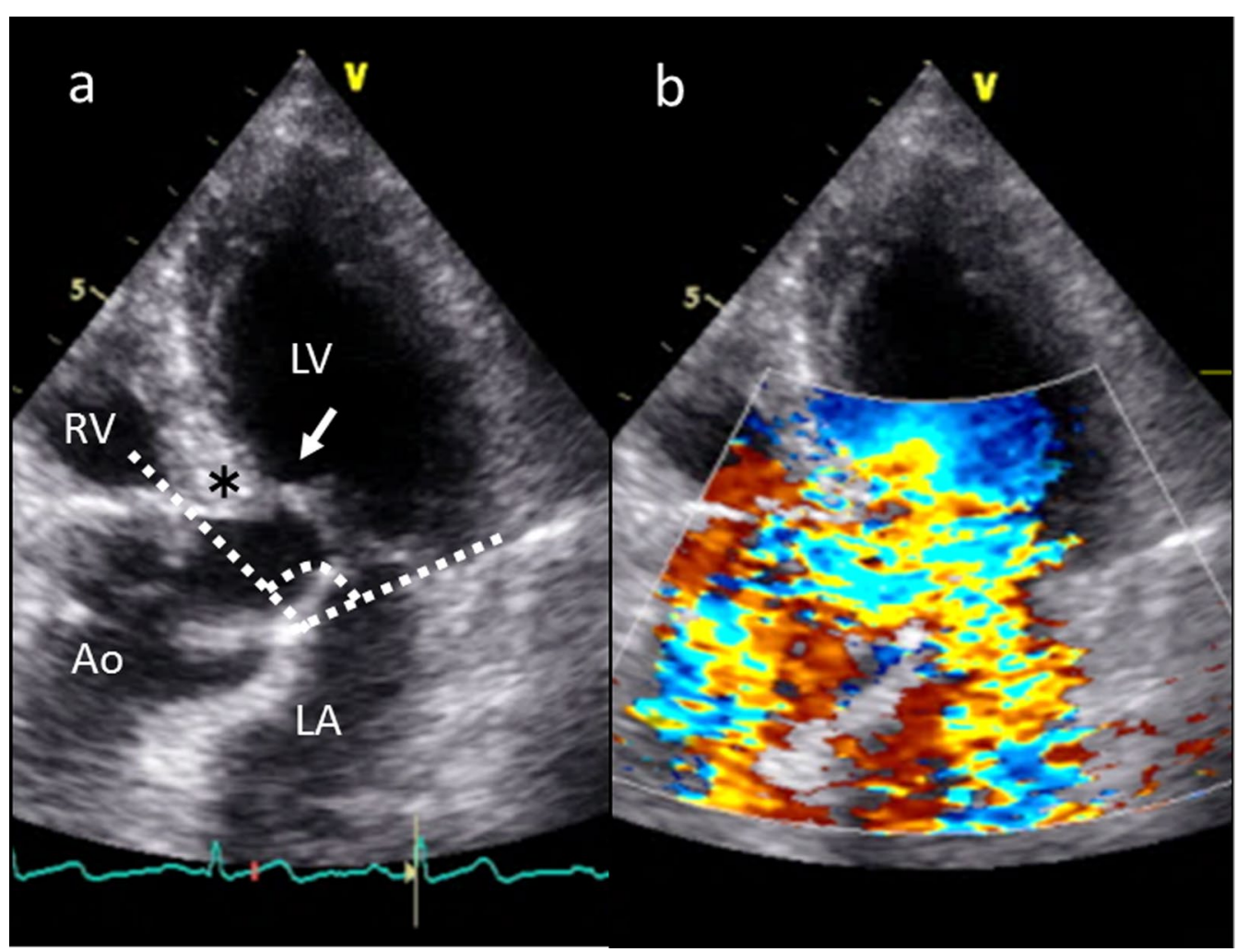

Fig. 2 Preoperative transthoracic echocardiographic views. a Left ventricular outflow tract obstruction with systolic anterior motion of the mitral valve (white arrow), thick basal interventricular septum, and septal bulging (black asterisk) due to narrow aorto-mitral angle (dotted lines). b Color Doppler imaging of mitral regurgitation and flow acceleration in the left ventricular outflow tract. Ao: aorta; LA: left atrium; LV: left ventricle; RV: right ventricle

beta-blocker therapy (landiolol hydrochloride $2 \mu \mathrm{g} / \mathrm{kg} /$ min) and infusion load.

The aortic false lumen was still thrombosed, but the ascending aortic maximum short diameter was dilated from 39 to $45 \mathrm{~mm}$ and the maximum thickness of the thrombosed false lumen increased from 4 to $8 \mathrm{~mm}$ in the preoperative CT scan at 14 days after the onset of aortic dissection. In case of postoperative unstable hemodynamics following the physical stressful aortic repair, concomitant IVSM during the ascending aortic replacement was planned for the prevention of SAM. The patient's adrenal insufficiency recovered gradually with alternate-day oral corticosteroid (prednisolone $5 \mathrm{mg}$ per dose); therefore, we performed the operation 19 days after the onset of aortic dissection. In the operating room, transesophageal echocardiography under total anesthesia still showed SAM. Following median sternotomy, IVSM and ascending aortic replacement with a one-branched J Graft Shield Neo 26-mm Dacron graft (Japan Lifeline Inc., Tokyo, Japan) were performed under usual cardiopulmonary bypass with cannulation via the right femoral artery and right atrium. In the operative findings, there was no pericardial effusion that could compress the LV structure. During the IVSM procedure, the needle stick technique (NST) (Fig. 3a, b) was applied because of easy, quick, and protective exposure to the IVS muscle to be resected. Briefly, three 21 -gauge needles were inserted into the interventricular septum just below the aortic valve annulus beyond the far side of the septal bulge. One needle was inserted beneath the mid portion of the right coronary cusp to provide a right-side margin, the second was inserted beneath the commissure of the right and left coronary cusps to provide a left-side margin, and the last was inserted between the previous two as a guide for the thickness of the myectomy (Fig. 3a). The resected muscle weighed $278 \mathrm{mg}$ in total (Fig. 3c). The cardiopulmonary bypass was easily weaned. Postoperative TTE showed improved systolic flow velocity in the LVOT of $0.93 \mathrm{~m} / \mathrm{sec}$ and no evidence of SAM (Fig. 4a, b). The patient was discharged from our hospital 22 days after the operation, with no major complications. 

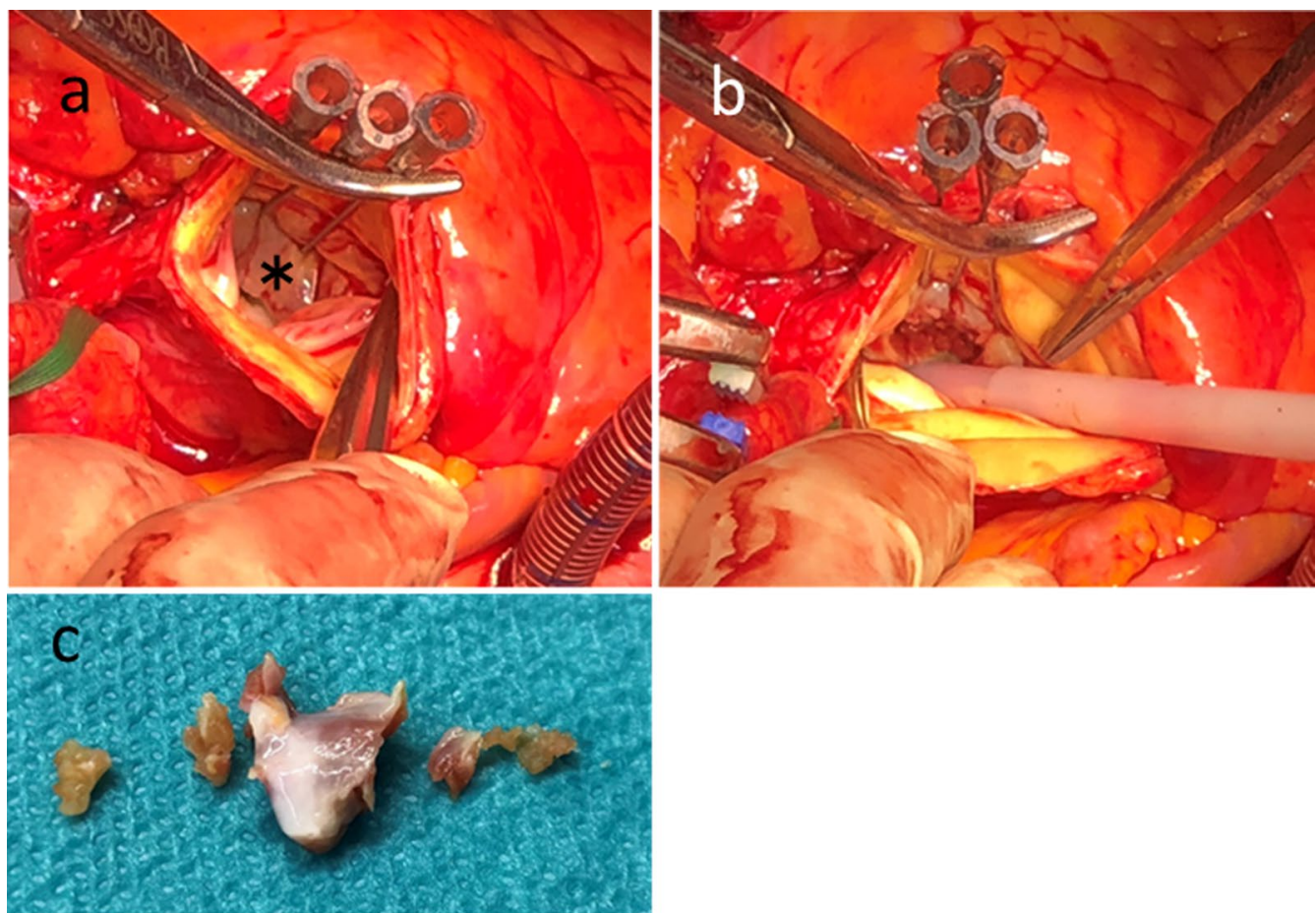

Fig. 3 Needle stick technique of the interventricular septal muscle resection. a Exposure of the part of the interventricular muscle to be resected. Black asterisk indicates the interventricular septal bulge. $\mathbf{b}$ Resected part of the interventricular septal muscle. $\mathbf{c}$ Resected muscle pieces of 278 mg in total

\section{Discussion}

AAD is occasionally complicated by aortic and vascular events, such as aortic valve regurgitation, aortic rupture, and malperfusion, but cases of SAM complicating AAD have seldom been described [3]. The present case had unstable SAM induced anatomically and hemodynamically by AAD. The concomitant IVSM procedure, performed with NST during the ascending aortic replacement, was conducted to prevent unstable hemodynamics during postoperative management.

SAM is a cardiac functional disorder to be avoided in perioperative cardiovascular management. SAM can be induced by dynamic interactions between cardiac anatomical features and hemodynamic conditions. The main mechanism of SAM has been recognized as the Venturi effect in the LVOT, and several cardiac anatomical features are known to affect the manifestation of SAM. SAM is most commonly seen in hypertrophic obstructive cardiomyopathy (HOCM). Structural abnormalities leading to SAM include LVH, a small LV cavity, IVS bulging due to a narrow aorto-mitral angle $\left(<120^{\circ}\right)$, and a mismatch between the amount of mitral valvular tissue and the area of the mitral valve orifice [1-4]. Hemodynamic conditions leading to SAM include low systemic arterial resistance, hypovolemia, tachycardia, a hyperdynamic left ventricle, and cardiac tamponade [3, 4]. In addition, SAM may occur after aortic valve replacement and repair and mitral valve procedures such as mitral repair because of a discrepancy between the amount of leaflet tissue and the size of the annular ring selected in mitral annuloplasty $[1,2]$. The anterior leaflet of the mitral valve exists on the LVOT in which the cardiac hemodynamics are finally concentrated. Therefore, SAM is considered a multifactorial cardiac functional disorder induced by the coexistence of several anatomical and hemodynamic components. The present case had, by chance, an anatomical feature of IVS bulging due to a narrow aortomitral angle before AAD. This is confirmed through a narrow aorto-mitral angle having been detected in her past CT images before the onset of AAD (Fig. 5a, b). Moreover, her medical history included rheumatoid arthritis controlled with oral corticosteroid medication. The SAM in the present case may have been affected by the patient's specific cardiac structure of IVS bulging due to a narrow aorto-mitral angle, coupled with lower cardiac afterload due to blood pressure control to prevent recurrent aortic events, and hypovolemia due to the relative adrenal failure following aortic dissection (Fig. 6). Stable hemodynamics were not considered to be maintained by ascending aortic replacement alone because of 


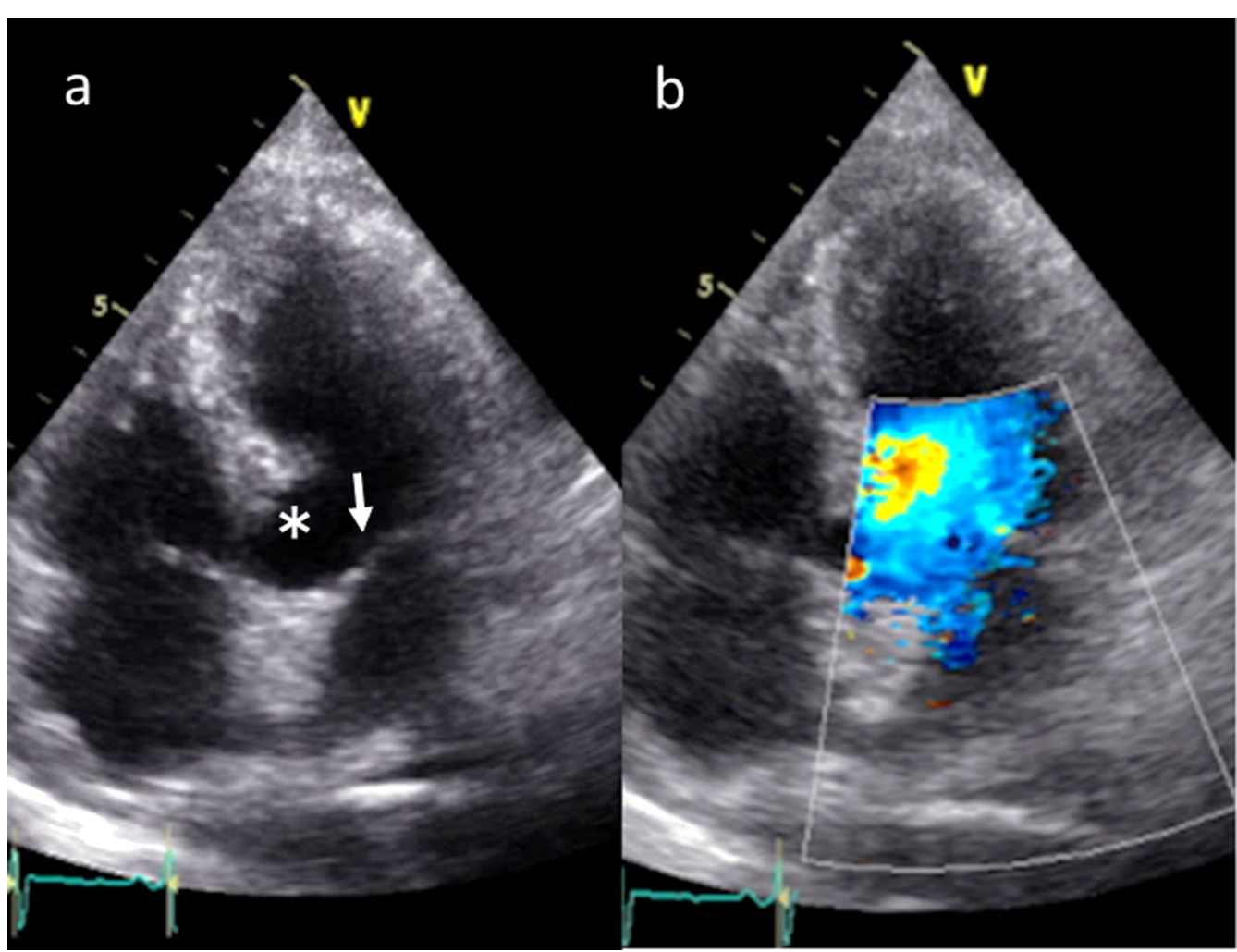

Fig. 4 Postoperative transthoracic echocardiographic views. a No evidence of systolic anterior motion (white arrow) and resected part of the basal interventricular septum (white asterisk). b Color Doppler imaging of no mitral regurgitation

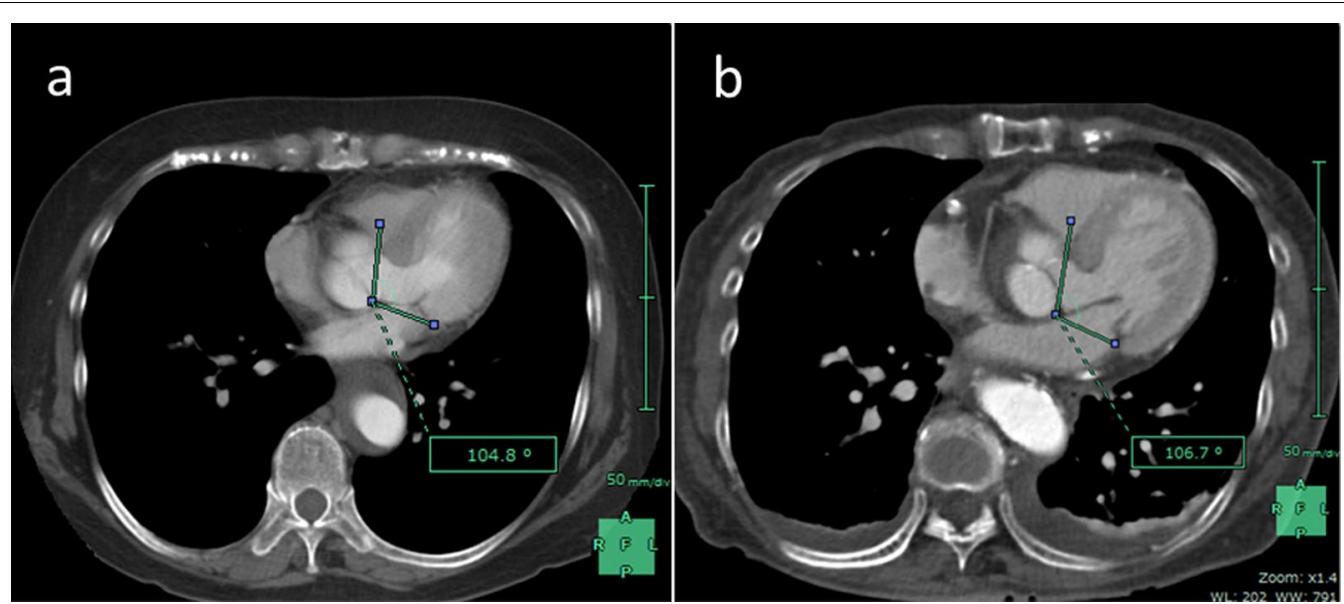

Fig. 5 Narrow aorto-mitral angle detected in computed tomography. a Her past image before the onset of acute type A aortic dissection (at the onset of type B aortic dissection). b Preoperative image after the onset of acute type A aortic dissection. Both images have almost the same cross section

reversible SAM in postoperative management. Therefore, concomitant IVSM during ascending aortic replacement was performed.
IVSM originates from the Morrow operation, which can diminish the Venturi effect with the resected IVS muscle in the LVOT [6]. IVSM has been conducted for the treatment of $\operatorname{HOCM}[6,7]$, sigmoid or bulging 


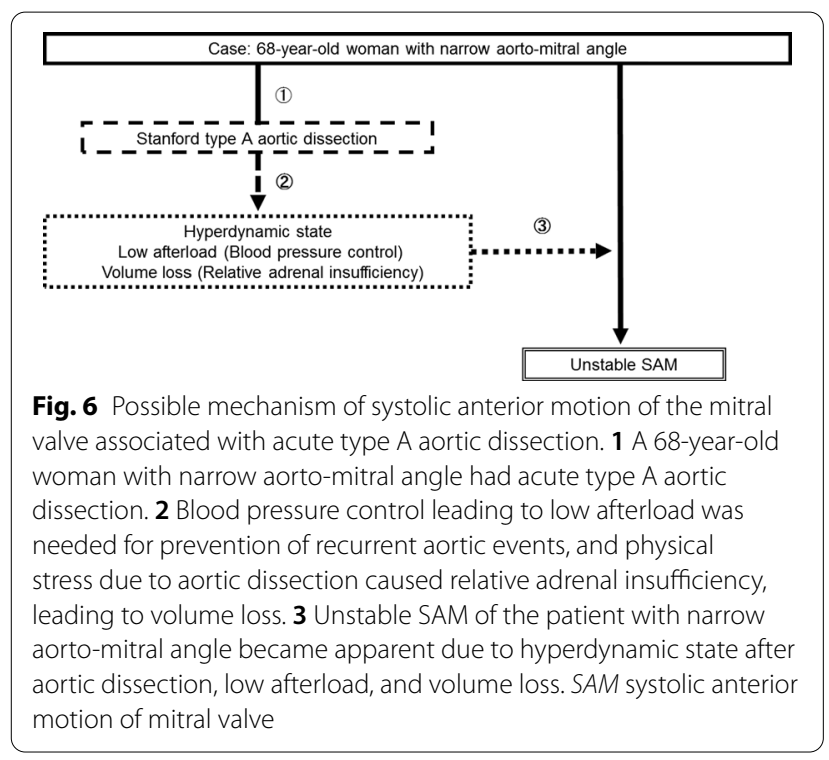

septum, and LVH due to aortic stenosis and hypertension. Indeed, IVSM in itself has good clinical results; however, there are several possible methods for appropriately exposing the IVS muscle to be resected. Usui and colleagues developed the NST in the resection procedure of the IVS muscle and described a report of 17 cases other than aortic dissection [7]. The NST enables surgeons to get a good exposure of the IVS muscle and grasp the part of the muscle to be resected three-dimensionally. Unlike the aortic retractor, which carries the risk of aortic intimal injuries, the NST is helpful for surgeons to safely achieve sufficient IVSM even in the case of AAD.

\section{Conclusions}

This report highlights the following points: (1) We presented a rare case of SAM associated with AAD that was cured by concomitant thoracic aortic replacement and IVSM. (2) We advocate the novel application of IVSM using the NST to cases of AAD. This is a safe and feasible procedure to prevent new intraoperative iatrogenic tears of the aortic intima and postoperative unstable SAM, even in the context of AAD.

\section{Abbreviations}

SAM: Systolic anterior motion; LV: Left ventricular; AAD: Acute type A aortic dissection; IVSM: Interventricular septal myectomy; CT: Computed tomography; TTE: Transthoracic echocardiography; LVOT: Left ventricular outflow tract; LVH: Left ventricular hypertrophy; IVS: Interventricular septal; NST: Needle stick technique; HOCM: Hypertrophic obstructive cardiomyopathy.

\section{Acknowledgements}

We would like to thank Editage (http://www.editage.com) for English language editing.

\section{Authors' contributions}

NY prepared the manuscript under the supervision of KO. NY and KO performed the operations and perioperative management. Both authors read and approved the final manuscript.

\section{Funding}

The authors declare that they received no financial support.

\section{Availability of data and materials}

None.

\section{Declarations}

Ethics approval and consent to participate.

The patient has provided permission to publish the features of her case, and her identity has been protected.

\section{Consent for publication}

Informed consent was obtained from the patient for the publication of this case report and any accompanying images.

\section{Competing interests}

The authors declare that they have no competing interests.

Received: 3 June 2021 Accepted: 9 August 2021

Published online: 16 August 2021

\section{References}

1. Carpentier A, Adams DH, Filsoufi F. Techniques in systolic anterior leaflet motion. In: Fletcher J, editor. Carpentier's reconstructive valve surgery from valve analysis to valve reconstruction. Maryland Heights, Missouri, MO, USA: Saunders/Elsevier; 2010. p. 157-65.

2. Alfieri $O$, Lapenna E. Systolic anterior motion after mitral valve repair: where do we stand in 2015? Eur J Cardiothorac Surg. 2015:48:344-6.

3. Park KS, Kim H, Jung YS, Kim HJ, Lee JM, Hong DM, et al. Left ventricular outflow tract obstruction with systolic anterior motion of the mitral valve in patient with pericardial effusion caused by ascending aortic dissection. Korean J Anesthesiol. 2013:64:73-6.

4. Kim K, Toyota T, Fujii Y, Kitai T, Kobori A, Ehara N, et al. Reversible severe mitral regurgitation caused by systolic anterior motion of the mitral valve in the absence of left ventricular hypertrophy: a case report. J Cardiol Cases. 2016:13:42-4.

5. Tagliari AP, Gavazzoni M, Miura M, Taramasso M, Maisano F. SAM and severe mitral regurgitation post-acute type A aortic dissection surgery treated with MitraClip. J Am Coll Cardiol Case Rep. 2020;2:1582-6.

6. Morrow AF. Hypertrophic subaortic stenosis. Operative methods utilized to relieve left ventricular outflow obstruction. J Thorac Cardiovasc Surg. 1978;76:423-30.

7. Usui A, Araki Y, Oshima H, Ueda Y. A needle stick technique for septal myectomy for hypertrophic obstructive cardiomyopathy. Ann Thorac Surg. 2013;95:726-8.

\section{Publisher's Note}

Springer Nature remains neutral with regard to jurisdictional claims in published maps and institutional affiliations. 